\title{
O uso de gírias: crenças, preconceitos e identidades
}

\author{
The use of Slangs: beliefs, prejudices and identities
}

\author{
Fernanda Cristina Viana \\ Universidade Estadual de Londrina \\ Jéssica Brandet Alves \\ Universidade Estadual de Londrina
}

\begin{abstract}
- RESUMO: A presente pesquisa visa analisar o que as gírias podem revelar do comportamento de alguns grupos sociais e de que maneira elas reafirmam sua identidade. Além disso, será analisado como o uso de gírias se realiza entre os grupos sociais, como pode identificar opiniões, crenças e preconceitos, além de compreender de que maneira as crenças sobre o uso de gírias podem afetar no comportamento social e linguístico dos informantes. Como instrumento de coleta de dados, foi aplicado um questionário em que, com os resultados obtidos, foram analisados os tópicos citados. Como resultados da pesquisa, os informantes apresentaram grande reprovação em relação ao uso de gírias, especialmente por mulheres, mostrando assim que as crenças dos falantes se manifestam sim por meio da linguagem.
\end{abstract}

- PAlAVRAS-CHAVE: Gírias. Crenças e preconceito. Grupos sociais.

- ABSTRACT: Analyze what slangs can reveal of some social groups behavior and how slangs reaffirm the identity of the group is the aim of this paper. Specifically, the analysis will comprehend how the use of slangs takes place among social groups, how slangs can identify opinions, beliefs, prejudices and how slangs can affect the informants' social and linguistic behavior. For collecting data, it was applied a form. The results were analyzed concerning the topics of the research. The result was a strong disapproval of slangs, especially by women. The speakers' beliefs are really manifested by the language use.

- KEYWORDS: Slangs. Beliefs and Prejudice. Social groups.

\footnotetext{
“(...) quanto maior for o sentimento de união que liga os membros do pequeno grupo, tanto mais a linguagem gíria servirá como elemento identificador" (VALADARES, 2011, p. 29).
}

\section{Introdução}

Gíria: Vocabulário momentâneo e novo, ou antigo que passa a possuir novos sentidos, usado por grupos específicos. Maneira de falar repleta de expressões próprias, gírias, palavras de uso restrito a grupos sociais. Modo de falar marginal, praticamente impossível de ser entendido por outras pessoas, que estabelece uma relação de pertencimento com o grupo. (DICIONÁRIO ONLINE DE PORTUGUÊS) 
As gírias são conhecidas como um acontecimento da linguagem que pode ser utilizado em diversas classes sociais, em um contexto informal independentemente da idade. São utilizadas no cotidiano por crianças, jovens e adultos e podem ser consideradas como um dialeto. São criadas com objetivo de substituir os termos formais que são usados na língua no dia-a-dia, com a intenção de facilitar a interpretação da expressão usada, porém, não devem ser interpretadas de maneira literal e sim no sentido figurado.

Geralmente são criadas por grupos sociais distintos, o que pode, muitas vezes, causar a incompreensão de outros grupos. A importância presente nessa forma de linguagem é exatamente a identidade que cada um pode carregar com os termos criados. Um exemplo disso são as gírias criadas por grupos marginalizados com a finalidade de não serem compreendidos pela sociedade em geral. Como afirma Flavio Biasutti Valadares (2011), citando Preti, em seu artigo intitulado Revisando a noção de gírias: do conceito à dicionarização:

\begin{abstract}
Nos termos de Preti (1984), o aparecimento da gíria como um fenômeno restrito é decorrente da dinâmica social e linguística inerente às línguas. Ainda é destacado pelo autor que ela é caracterizada como um vocabulário especial, sendo considerada um signo de grupo, a princípio secreto, de domínio exclusivo de uma comunidade social restrita. O linguista ressalta que, quanto maior for o sentimento de união que liga os membros do pequeno grupo, tanto mais a linguagem gíria servirá como elemento identificador, diferenciando o falante na sociedade e servindo como meio ideal de comunicação, além de forma de autoafirmação (2011, p. 29).
\end{abstract}

Outra maneira de se criar uma gíria é por influência de modismos que são apresentados por meio de veículos de comunicação como a internet, rádio, TV, entre outros. As pessoas não familiarizadas com esse tipo de expressão podem encontrar dificuldades de entendimento em uma conversa informal, como em algumas expressões são encontradas facilmente em nosso dia-a-dia, como, por exemplo:

- Partiu: é a gíria usada para transmitir a ideia de sair imediatamente, no sentido de "vamos lá" ou "vamos agora".

- Biscoiteiro (a): termo utilizado em forma de deboche para identificar pessoas que fazem de tudo para chamar a atenção e receber elogios de outras.

- Cremosa: é usado quando alguém deseja insinuar que alguma garota é bonita, sexy ou atraente. Pode ser usado no masculino também.

- Fada sensata: gíria utilizada para se referir a mulheres com conhecimento sobre determinados assuntos com o objetivo de afirmar que elas estão corretas sobre o que dizem.

Por meio deste trabalho, busca-se analisar o que as gírias podem revelar do comportamento de alguns grupos sociais e de que maneira elas reafirmam a identidade deles. As gírias podem ser consideradas um dialeto, fazendo assim parte dos estudos de variação linguística, porém é importante diferenciá-las de outros estudos dessa área como jargões, regionalismos, coloquialismo, entre outros, pois:

Isso provoca uma generalização deste conceito, ocasionando certa confusão nos usuários da língua. Entretanto, conforme observado nos verbetes dos dicionários, as gírias são espécies de "códigos secretos" para um determinado grupo manter interações. Nesse sentido, há uma grande diferença entre gírias e regionalismos, por exemplo, uma vez que estes estão demarcados por regiões linguístico-geográficas e aquelas não. [...]. Evidentemente, uma gíria pode 
também ser um regionalismo, não há impedimento; contudo, os sentidos construídos e os objetivos do seu uso, com certeza, serão diferentes (VALADARES, 2011, p. 30-31).

O que será analisado em relação às gírias não são apenas seus significados, mas também o seu papel formador de identidade das pessoas que fazem uso delas. É fácil notar que as gírias variam de um grupo social para o outro. Nesta análise será feita a abordagem de como essa variação se dá e quais são os possíveis elementos causadores dela.

Sabe-se que uma língua tem em si a carga histórica e social do povo que a usa, dessa maneira ela é determinante para que se possa entender o comportamento da sociedade. As variações linguísticas revelam traços da identidade social dos indivíduos e as gírias também fazem parte disso. Dessa maneira, seu estudo torna-se fundamental para a compreensão das mudanças, não apenas linguísticas, mas também do comportamento da sociedade.

Outro fator que torna o estudo das gírias essencial é o fato de que, conforme criamse novas gírias, elas acabam por substituir as antigas, pois, conforme Travaglia (2003), a língua se modifica com o passar do tempo. Assim, pode-se afirmar que as gírias tem a possibilidade de se sobrepor às palavras que as originam, tornando-se formas oficiais em determinado momento se forem aceitas pela sociedade em geral, como afirma Valadares:

[...] uma variação linguística pressupõe valor social, ou seja, variantes empregadas por falantes de estratos mais baixos da população em grande parte são estigmatizadas; todavia, à proporção que a variante passa a ser usada por grupos, o estigma vai diminuindo até deixar de existir completamente, se aceita pela classe dominante (2011, p. 29).

Elas também, em alguns casos, acabam sendo oficializadas por dicionários, como por exemplo, o termo mano que está presente no dicionário Aurélio: "Ma.no sm. Fam. 1. Irmão (1) 2. Amigo cordial (2)” (2008, p. 535).

Além dos fatores citados anteriormente, o preconceito, linguístico ou social pode ficar evidente nas crenças que os indivíduos possuem sobre as gírias. Dessa maneira, este trabalho tentará compreender esses pensamentos sobre o uso de gírias para a colaboração da desconstrução desses preconceitos e paradigmas, abordando essas gírias e defendendo o fato de que elas também fazem parte da Língua Portuguesa.

Dessa maneira, o objetivo geral desta pesquisa é analisar o que as gírias podem revelar do comportamento de alguns grupos sociais e de que maneira elas reafirmam sua identidade. Também será analisado como o uso de gírias se realiza entre os grupos sociais. Outros objetivos são identificar opiniões, crenças e preconceitos sobre o uso de gírias e compreender de que maneira as crenças sobre o uso de gírias podem afetar no comportamento social e linguístico dos informantes.

\section{A gíria como estudo e as crenças e atitudes linguísticas dos falantes}

Guedelha (2011), sobre crenças e atitudes linguísticas afirma que há nos falantes estereótipos em relação aos diferentes sotaques brasileiros, vendo-os como um vício. $\mathrm{O}$ autor entrevistou informantes de três capitais brasileiras: São Luís (Ma), Belém (Pa) e Manaus (Am) e confirmou que as crenças linguísticas envolvem estereótipos e preconceitos historicamente situados.

Guedelha apresenta a ideologia de Calvet (2002) que mostra que a relação do falante e sua língua nunca é neutra. Na própria língua e nas variedades linguísticas estão presentes os sentimentos e ideologias dos falantes e, dessa maneira, as pessoas acabam 
sendo julgadas pelo seu modo de falar, nascendo assim uma série de preconceitos e estereótipos em relação à língua.

Guedelha afirma também que a língua não existe sem os seus falantes, pois são eles que criam novos dialetos e os colocam em uso. $\mathrm{O}$ autor traz a ideia que Brandão (1991) tem sobre o tema "A esse respeito, Brandão (1991), por exemplo, enfatiza o duplo poder que o usuário de uma língua tem sobre ela: de utilizá-la e de modificá-la no decurso dessa utilização, acrescendo-lhe novos matizes a cada nova situação". Isso confirma o fato de que é o falante quem modifica a língua ao longo do tempo e é preciso aceitar a existência da variação linguística como uma realidade irreversível. Guedelha ainda afirma:

[...] só é possível se conhecer de fato um determinado grupo humano debruçando-se sobre a(s) forma(s) como esse grupo representa a sua história, os seus costumes e o ambiente em que vive. Assim sendo, procurar estudar essa história, costumes ou ambiente, sem proceder a uma acurada pesquisa linguística, seria desperdiçar tempo e deixar de lado um inestimável tesouro necessário a um conhecimento mais completo e significativo dessa comunidade. (GUEDELHA, 2011, p. $06)$.

O autor defende que encarar a ideia de que a língua é um sistema invariável significa negar a sua própria natureza de variedades, pois não existe uma pureza da língua, ela pode conter variações que, muitas vezes, alguns afirmam que não existiam antigamente.

Em outro estudo, Valadares (2011), discute a dicionarização e traz conceitos de gírias defendidos por ele e por outros teóricos como Preti. O autor também traz reflexões a cerca das gírias como variação linguística e as reconhece como um dialeto sociocultural, diferenciando-as de regionalismos e coloquialismos. Valadares ainda aborda a variação linguística citando autores como Travaglia (2003), Labov (2008) e Herzog (2006).

$\mathrm{O}$ autor apresenta em seu trabalho exemplos de gírias já dicionarizadas e aponta que essas gírias desempenham um papel importante na Língua Portuguesa como qualquer outra variação linguística, pois, segundo o autor, elas também são responsáveis pelas mudanças e renovações do seu léxico.

Aguilera (2008), sobre as crenças e atitudes linguísticas dos falantes das capitais brasileiras estabelece discussões acerca das crenças e atitudes linguísticas que informantes urbanos de capitais brasileiras realizam e assumem em sua fala.

A autora também destaca em seu trabalho a importância dos estudos sobre essas crenças e atitudes linguísticas que, segundo ela, são muito defendidos pela sociolingüística, porém acabam por não ser muito explorados. Dessa maneira, considera a variação linguística como fator definidor da identidade de grupos sociais que a assume e aponta componentes da atitude linguística utilizando os estudos de Lambert (1967) e Goméz Molina (1987). No artigo, também estão presentes os relatos de informantes sobre suas próprias crenças e atitudes em relação à língua.

\section{Metodologia}

Para a realização deste trabalho, foi elaborado um questionário sobre o assunto que os informantes responderam. Foram utilizados para isso gravadores eletrônicos e anotações, pois segundo Bortoni-Ricardo (2008, p.62) "a gravação eletrônica em vídeo ou áudio tem uma grande vantagem na coleta de dados porque permite ao observador "revisitar" os dados muitas vezes para tirar dúvidas e refinar a teoria que está construindo". 
Em seguida, foi realizada a análise dos dados seguindo a fundamentação teórica, usando como base os estudos de Guedelha (2011), Valadares (2011) e Aguilera (2008). Os informantes são do sexo masculino e feminino, de faixa etária entre 18 e 30 anos. A quantidade de informantes consiste no total de 36 entrevistados, sendo eles dois homens e duas mulheres de cada centro da Universidade Estadual de Londrina.

A pesquisa de campo, com informantes dos diferentes centros da Universidade Estadual de Londrina (UEL) - CCA, CLCH, CESA, CECA, CCB, CCE, CTU, CCS E CEFE -, teve o objetivo de compreender o que esses informantes pensam do uso de gírias e como o configuram em seu dia-a-dia.

As perguntas feitas aos informantes são:

1. Como você se refere a alguém que acha bonito (a) em contexto formal?

2. Como você se refere a alguém que acha bonito (a) em contexto informal?

3. Você ainda utiliza as mesmas gírias de sua adolescência?

4. Qual a sua opinião sobre o uso de gírias por homens? E por mulheres?

5. Para você, existe diferença entre gírias masculinas e femininas?

\section{As percepções dos grupos sociais quanto ao uso de gírias}

A pesquisa feita a partir do questionário apontou que as gírias ainda são vistas com preconceito por alguns grupos sociais. Quatorze dos trinta e seis informantes afirmaram que o uso de gírias, em sua opinião, é algo "feio", "desnecessário", "chato" e "estranho".

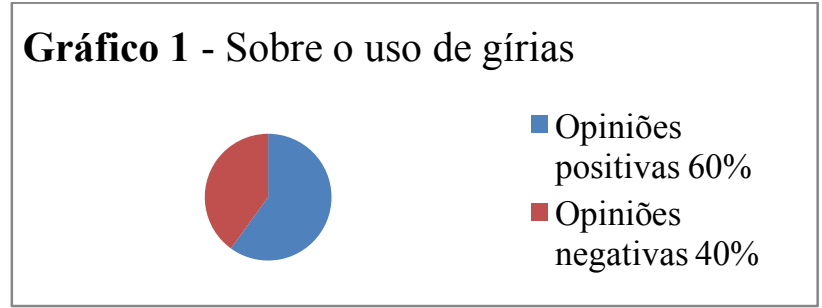

Quando questionados acerca da opinião sobre o uso de gírias por homens e por mulheres, vinte e cinco dos entrevistados acreditam que as mulheres não deveriam usar gírias, pois é "vulgar", "feio" e "estranho", sendo que nove desses mesmos informantes afirmaram ser perfeitamente normal que homens usem gírias.

\begin{tabular}{|l} 
Gráfico 2 - Sobre o uso de gírias \\
por mulheres \\
\\
apiniões \\
Positivas - \\
$25 \%$ \\
Opiniões \\
\hline
\end{tabular}

Neste ponto é possível ver a crença sobre a língua desses informantes se manifestar, mostrando uma atitude de reprovação diante da fala feminina.

Ao questionar se os informantes ainda utilizavam as gírias de sua adolescência, apenas quinze deles responderam que sim. Vinte e um dos trinta e seis informantes disseram não utilizar mais, sendo que cinco deles afirmaram que nunca utilizaram gírias. Esses cinco estavam dentro da quantidade de informantes que responderam que o uso de gírias é algo "feio". 
Gráfico 3 - Ainda utilizam as

gírias de sua adolescência?

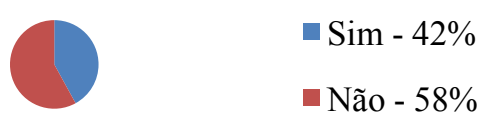

Para outro ponto abordado - diferenças entre gírias masculinas e femininas dezoito dos informantes afirmaram que acreditam que existem diferenças. É importante ressaltar que os outros dezoito informantes, que acreditam não haver diferenças, consistem no grupo mais jovem entrevistado, sendo eles da idade de18 até 25 anos.

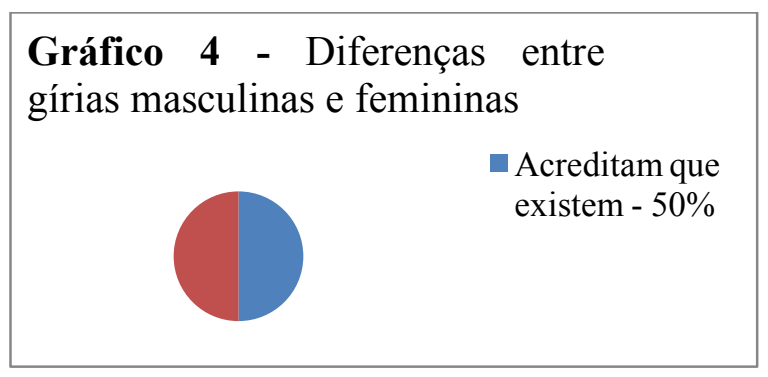

\subsection{O que os resultados podem revelar?}

A partir das respostas concedidas pelos informantes, pode-se concluir que, apesar das gírias serem algo extremamente comum atualmente, para uma boa parte dos informantes ela ainda é vista como algo que causa estranheza e que deve ser evitado.

Também é possível notar que o "machismo" ainda está enraizado na sociedade e pode-se perceber isso até mesmo na linguagem, já que uma parte dos informantes acredita que as mulheres não devem utilizar gírias enquanto, para esses mesmos informantes, o uso de gírias pelos homens é visto como algo perfeitamente normal.

A idade é outro fator que interfere no uso de gírias. A maioria dos informantes afirmou que não fazem mais uso de gírias que costumavam falar na adolescência, mostrando que as experiências de vida podem interferir na maneira com que o falante se comunica socialmente. $O$ caráter de imaturidade que as gírias possuem pode estar relacionados a essas respostas dadas pelos informantes, pois elas são vistas como parte da linguagem jovem, sendo seu uso algo a ser "superado" com o amadurecimento.

Além disso, os informantes mais jovens mostraram acreditar que tanto homens quanto mulheres fazem uso das mesmas gírias. Acerca disso, pode-se tirar algumas conclusões.

É possível dizer que as mudanças sociais e a luta por igualdade de gênero podem ser fatores determinantes no uso da linguagem. Também se pode afirmar que o uso de gírias vem passando por mudanças, caminhando para não ser mais visto com normalidade apenas quando colocado em prática pelo sexo masculino.

Outro ponto interessante na pesquisa é, que apesar de muitos dos informantes não terem opiniões positivas em relação ao uso de gírias, muitos deles, quando questionados sobre como se referem a alguém que acham bonito em situação informal, responderam que usam algum termo classificado como gíria. Um exemplo disso é o informante masculino 2 do CTU que afirmou não gostar de gírias, porém faz uso do termo "gata para caramba" ao se referir a alguém que considera bonito em contexto informal. 


\section{Considerações finais}

As gírias estão cada vez mais presentes no vocabulário dos falantes. Elas fazem parte das variações linguísticas e seu uso pode revelar pensamentos, crenças e preconceitos enraizados nos falantes de Língua Portuguesa. Uma maneira de ser perceber isso é por meio do estudo do que pensam os grupos sociais acerca disso, pois

[...] as atitudes linguísticas - e os preconceitos oriundos delas - são voltados não para aspectos relacionados à língua propriamente dita, mas a aspectos social e culturalmente instituídos pela sociedade (PATRIOTA, p. 3354).

Dessa maneira, este trabalho conclui que as gírias podem revelar do comportamento de alguns grupos sociais e de que maneira elas reafirmam a identidade deles, revelando quais são suas crenças e preconceitos diante deste objeto.

A partir dos exemplos analisados, percebeu-se que o uso de gírias ainda é subestimado pela sociedade, visto, muitas vezes, é tido como algo feio e que deve ser evitado. Isso representa que ainda há preconceito em relação ao uso de gírias.

Também se pôde revelar que o machismo ainda está enraizado na sociedade, sendo a opinião sobre o uso de gírias por mulheres - visto com maus olhos por uma parte dos informantes - uma maneira de identificá-lo. Assim, é possível concluir que o comportamento linguístico revela fatores sociais e pode ser utilizado como meio de abordá-los e discutir acerca deles.

\section{REFERÊNCIAS}

AGUILERA, Vanderci de Andrade. Crenças e atitudes linguísticas: o que dizem os falantes das capitais brasileiras. Estudos Linguísticos, São Paulo, 37 (2), maio-ago. 2008, p. 105-112.

BORTONI-RICARDO, Stella Maris. Postulados do Paradigma Interpretativista. In: O professor pesquisador: introdução à pesquisa qualitativa. São Paulo: Parábola, 2008, p.31 a 40 .

FERREIRA, Aurélio Buarque de Holanda. Míni Aurélio: o minidicionário da Língua Portuguesa. 7 ed. - Curitiba: Ed Positivo; 2008.

GUEDELHA, Carlos Antônio Magalhães. Crenças e atitudes linguísticas: um estudo dialetológico.

Disponível

em: http://www.ufjf.br/revistagatilho/files/2011/10/guedelha.pdf. Acesso em: 25/05/2019.

PADILHA, Adriano. Gírias atuais mais usadas na internet. Disponível em: https://www.dicionariopopular.com/girias-atuais-internet/. Acesso em: 23/05/2019.

PATRIOTA, Luciene Maria. Atitudes Linguísticas frente às Gírias: O Preconceito. UFCG: XI Encontro Latino de Iniciação Científica e VII Encontro Latino Americano de Pós-Graduação.

PEREZ, Luana Castro Alves. Dialetos e registro. Disponível em: https://www.portugues.com.br/redacao/dialetos-registros.html. Acesso em: 24/05/2019.

PORTUGUÊS, Dicionário Online. Disponível em: https://www.dicio.com.br/giria/. Acesso em: 10/09/2019

TRAVAGLIA, L. C. Gramática e interação: uma proposta para o ensino de gramática. São Paulo: Cortez, 2003. 
VALADARES. Flavio Biasutti. Revisando a noção de gírias: do conceito à dicionarização. Revista Eletrônica de Linguística, vol 5, nº 1; 2011, p. 27-43.

VILARINHO, Sabrina. Gíria. Disponível em:

https://mundoeducacao.bol.uol.com.br/redacao/giria-1.htm. Acesso em: 10/09/2019.

Anexo:

Quadros de respostas das pesquisas com os informantes:

1. Como você se refere a alguém que acha bonito (a) em contexto formal?

\begin{tabular}{|l|l|l|l|}
\hline Centro & Sexo & Idade & Resposta \\
\hline CLCH & $\mathrm{M}$ & 19 & Bela \\
\hline CLCH & $\mathrm{M}$ & 18 & Bonita \\
\hline CLCH & $\mathrm{F}$ & 18 & Lindo \\
\hline CLCH & $\mathrm{F}$ & 26 & Elegante \\
\hline CCA & $\mathrm{M}$ & 28 & Linda \\
\hline CCA & $\mathrm{M}$ & 26 & Interessante \\
\hline CCA & $\mathrm{F}$ & 25 & Bonito \\
\hline CCA & $\mathrm{F}$ & 22 & Bonito \\
\hline CESA & $\mathrm{M}$ & 30 & Bonita \\
\hline CESA & $\mathrm{M}$ & 25 & Bonita \\
\hline CESA & $\mathrm{F}$ & 25 & Interessante \\
\hline CESA & $\mathrm{F}$ & 25 & Bonito \\
\hline CECA & $\mathrm{M}$ & 18 & Lindíssima \\
\hline CECA & $\mathrm{M}$ & 21 & Maravilhosa \\
\hline CECA & $\mathrm{F}$ & 21 & Lindo \\
\hline CECA & $\mathrm{F}$ & 30 & Bonito \\
\hline CCB & $\mathrm{M}$ & 28 & Linda \\
\hline CCB & $\mathrm{M}$ & 21 & Bela \\
\hline
\end{tabular}

\begin{tabular}{|l|l|l|l|}
\hline CCB & $\mathrm{F}$ & 18 & Graciosa \\
\hline CCB & $\mathrm{F}$ & 24 & Interessante \\
\hline CCE & $\mathrm{M}$ & 23 & Bonita \\
\hline CCE & $\mathrm{M}$ & 24 & Bonita \\
\hline CCE & $\mathrm{F}$ & 22 & Charmoso \\
\hline CCE & $\mathrm{F}$ & 26 & Lindo \\
\hline CTU & $\mathrm{M}$ & 30 & Maravilhosa \\
\hline CTU & $\mathrm{M}$ & 30 & Formosa \\
\hline CTU & $\mathrm{F}$ & 28 & Bonito \\
\hline CTU & $\mathrm{F}$ & 24 & Lindo \\
\hline CCS & $\mathrm{M}$ & 30 & Bela \\
\hline CCS & $\mathrm{M}$ & 20 & Linda \\
\hline CCS & $\mathrm{F}$ & 27 & Bonito \\
\hline CCS & $\mathrm{F}$ & 26 & Belo \\
\hline CEFE & $\mathrm{M}$ & 27 & Linda \\
\hline CEFE & $\mathrm{M}$ & 30 & Esbelta \\
\hline CEFE & $\mathrm{F}$ & 30 & Lindo \\
\hline CEFE & $\mathrm{F}$ & 29 & Bonito \\
\hline
\end{tabular}

2. Como você se refere a alguém que acha bonito (a) em contexto informal?

\begin{tabular}{|l|l|l|l|}
\hline Centro & Sexo & Idade & Resposta \\
\hline CLCH & $\mathrm{M}$ & 19 & Thucthuca \\
\hline CLCH & $\mathrm{M}$ & 18 & Gostosinha \\
\hline CLCH & $\mathrm{F}$ & 18 & Gatinho \\
\hline CLCH & $\mathrm{F}$ & 26 & Chuchu \\
\hline CCA & $\mathrm{M}$ & 28 & Gata \\
\hline CCA & $\mathrm{M}$ & 26 & Gata \\
\hline CCA & $\mathrm{F}$ & 25 & Gostoso \\
\hline CCA & $\mathrm{F}$ & 22 & Delicioso \\
\hline CESA & $\mathrm{M}$ & 30 & Pitélzinho \\
\hline CESA & $\mathrm{M}$ & 25 & Gata \\
\hline CESA & $\mathrm{F}$ & 25 & Gatão \\
\hline CESA & $\mathrm{F}$ & 25 & Gato \\
\hline CECA & $\mathrm{M}$ & 18 & Carinho \\
\hline CECA & $\mathrm{M}$ & 21 & Gostosa \\
\hline CECA & $\mathrm{F}$ & 21 & Ô lá em casa \\
\hline CECA & $\mathrm{F}$ & 30 & Brotão \\
\hline CCB & $\mathrm{M}$ & 28 & Filé \\
\hline CCB & $\mathrm{M}$ & 21 & Filézinho \\
\hline
\end{tabular}

\begin{tabular}{|l|l|l|l|}
\hline CCB & F & 18 & Uvinha \\
\hline CCB & F & 24 & Pão \\
\hline CCE & M & 23 & Gata \\
\hline CCE & M & 24 & Gatinha \\
\hline CCE & F & 22 & Baita homem \\
\hline CCE & F & 26 & Varão, gato \\
\hline CTU & M & 30 & Deusa \\
\hline CTU & M & 30 & Gostosa \\
\hline CTU & F & 28 & Gato \\
\hline CTU & F & 24 & Filézão \\
\hline CCS & M & 30 & Gatinha \\
\hline CCS & M & 20 & Gata para caramba \\
\hline CCS & F & 27 & Delicinha \\
\hline CCS & F & 26 & Gatão \\
\hline CEFE & M & 27 & Espetáculo \\
\hline CEFE & M & 30 & Anjo \\
\hline CEFE & F & 30 & Gostosinho \\
\hline CEFE & F & 29 & Gato \\
\hline & & & \\
\hline
\end{tabular}

3. Você ainda utiliza as mesmas gírias de sua adolescência?

\begin{tabular}{|l|l|l|l|}
\hline Centro & Sexo & Idade & Resposta \\
\hline CLCH & M & 19 & Sim: pode crê \\
\hline CLCH & M & 18 & Sim \\
\hline CLCH & $\mathrm{F}$ & 18 & Sim \\
\hline CLCH & $\mathrm{F}$ & 26 & Sim: mano, é nóis \\
\hline CCA & M & 28 & Não \\
\hline CCA & M & 26 & Sim: mulecote, firmeza \\
\hline CCA & F & 25 & Não \\
\hline CCA & F & 22 & Não \\
\hline CESA & $M$ & 30 & Não \\
\hline CESA & $M$ & 25 & Não gosta \\
\hline CESA & F & 25 & Sim: putz griles \\
\hline
\end{tabular}

\begin{tabular}{|l|l|l|l|}
\hline CESA & $\mathrm{F}$ & 25 & Não \\
\hline CECA & $\mathrm{M}$ & 18 & Sempre \\
\hline CECA & $\mathrm{M}$ & 21 & Sim \\
\hline CECA & $\mathrm{F}$ & 21 & Com certeza \\
\hline CECA & $\mathrm{F}$ & 30 & Não \\
\hline CCB & $\mathrm{M}$ & 28 & Sim: demorou, falou \\
\hline CCB & $\mathrm{M}$ & 21 & Não \\
\hline CCB & $\mathrm{F}$ & 18 & Sim \\
\hline CCB & $\mathrm{F}$ & 24 & Sim \\
\hline CCE & $\mathrm{M}$ & 23 & Sim \\
\hline CCE & $\mathrm{M}$ & 24 & Não \\
\hline CCE & $\mathrm{F}$ & 22 & Não \\
\hline
\end{tabular}




\begin{tabular}{|l|l|l|l|}
\hline CCE & $\mathrm{F}$ & 26 & Não, nunca utilizou \\
\hline CTU & $\mathrm{M}$ & 30 & Não \\
\hline CTU & $\mathrm{M}$ & 30 & Não gosta de gírias \\
\hline CTU & $\mathrm{F}$ & 28 & Não \\
\hline CTU & $\mathrm{F}$ & 24 & Não, nunca usou \\
\hline CCS & $\mathrm{M}$ & 30 & Não \\
\hline CCS & $\mathrm{M}$ & 20 & Sim \\
\hline
\end{tabular}

\begin{tabular}{|l|l|l|l|}
\hline CCS & $\mathrm{F}$ & 27 & Não \\
\hline CCS & $\mathrm{F}$ & 26 & Não \\
\hline CEFE & $\mathrm{M}$ & 27 & Não \\
\hline CEFE & $\mathrm{M}$ & 30 & Não \\
\hline CEFE & $\mathrm{F}$ & 30 & Não \\
\hline CEFE & $\mathrm{F}$ & 29 & Não \\
\hline
\end{tabular}

4. Qual a sua opinião sobre o uso de gírias por homens? E por mulheres?

\begin{tabular}{|l|l|l|l|}
\hline Centro & Sexo & Idade & Resposta \\
\hline CLCH & M & 19 & Por ambos: legal \\
\hline CLCH & M & 18 & Por ambos: legal \\
\hline CLCH & F & 18 & Por ambos: muito normal \\
\hline CLCH & F & 26 & Por ambos: normal \\
\hline CCA & M & 28 & $\begin{array}{l}\text { Homem: normal } \\
\text { Mulher: feio }\end{array}$ \\
\hline CCA & M & 26 & Por ambos: feio \\
\hline CCA & F & 25 & Por ambos: feio \\
\hline CCA & F & 22 & Não \\
\hline CESA & M & 30 & $\begin{array}{l}\text { Homem: tranquilo } \\
\text { Mulher: estranho }\end{array}$ \\
\hline CESA & M & 25 & Por ambos: feio \\
\hline CESA & F & 25 & $\begin{array}{l}\text { Homem: normal } \\
\text { Mulher: vulgar }\end{array}$ \\
\hline CESA & F & 25 & Por ambos: feio \\
\hline CECA & M & 18 & Por ambos: normal \\
\hline CECA & M & 21 & $\begin{array}{l}\text { Homem: normal } \\
\text { Mulher: estranho }\end{array}$ \\
\hline CECA & F & 21 & Por ambos: super legal \\
\hline CECA & F & 30 & Por ambos: legal \\
\hline CCB & M & 28 & $\begin{array}{l}\text { Por ambos: depende da } \\
\text { situação }\end{array}$ \\
\hline CCB & M & 21 & $\begin{array}{l}\text { Homem: normal } \\
\text { Mulher: pesado }\end{array}$ \\
\hline CCB & F & 18 & $\begin{array}{l}\text { Por ambos: dependendo do } \\
\text { lugar é legal }\end{array}$ \\
\hline
\end{tabular}

\begin{tabular}{|l|l|l|l|}
\hline CCB & F & 24 & $\begin{array}{l}\text { Homem: normal } \\
\text { Mulher: estranho }\end{array}$ \\
\hline CCE & M & 23 & Por ambos: normal \\
\hline CCE & M & 24 & Por ambos: desnecessário \\
\hline CCE & F & 22 & $\begin{array}{l}\text { Por ambos: muito feio, não } \\
\text { gosta de gírias }\end{array}$ \\
\hline CCE & F & 26 & $\begin{array}{l}\text { Por ambos: extremamente } \\
\text { feio }\end{array}$ \\
\hline CTU & M & 30 & $\begin{array}{l}\text { Homem: normal } \\
\text { Mulher: vulgar }\end{array}$ \\
\hline CTU & M & 30 & Por ambos: muito feio \\
\hline CTU & F & 28 & $\begin{array}{l}\text { Homem: estranho; } \\
\text { Mulher: feio }\end{array}$ \\
\hline CTU & F & 24 & Por ambos: desagradável \\
\hline CCS & M & 30 & $\begin{array}{l}\text { Homem: normal } \\
\text { Mulher: feio }\end{array}$ \\
\hline CCS & M & 20 & Por ambos: indiferente \\
\hline CCS & F & 27 & $\begin{array}{l}\text { Homem: estranho } \\
\text { Mulher: muito feio }\end{array}$ \\
\hline CCS & F & 26 & $\begin{array}{l}\text { Homem: feio } \\
\text { Mulher: horrível }\end{array}$ \\
\hline CEFE & M & 27 & $\begin{array}{l}\text { Homem: chato } \\
\text { Mulher: pesado }\end{array}$ \\
\hline CEFE & M & 30 & Por ambos: desrespeitoso \\
\hline CEFE & F & 30 & Por ambos: estranho \\
\hline CEFE & F & 29 & $\begin{array}{l}\text { Homem: normal } \\
\text { Mulher: estranho }\end{array}$ \\
\hline
\end{tabular}

5. Para você, existe diferença entre gírias masculinas e femininas?

\begin{tabular}{|l|l|l|l|}
\hline Centro & Sexo & Idade & Resposta \\
\hline CLCH & $\mathrm{M}$ & 19 & Não \\
\hline CLCH & $\mathrm{M}$ & 18 & Não \\
\hline CLCH & $\mathrm{F}$ & 18 & Não \\
\hline CLCH & $\mathrm{F}$ & 26 & Não \\
\hline CCA & $\mathrm{M}$ & 28 & Sim \\
\hline CCA & $\mathrm{M}$ & 26 & Sim \\
\hline CCA & $\mathrm{F}$ & 25 & Sim \\
\hline CCA & $\mathrm{F}$ & 22 & Sim \\
\hline CESA & $\mathrm{M}$ & 30 & Sim \\
\hline CESA & $\mathrm{M}$ & 25 & Não \\
\hline CESA & $\mathrm{F}$ & 25 & Sim \\
\hline CESA & $\mathrm{F}$ & 25 & Sim \\
\hline CECA & $\mathrm{M}$ & 18 & Não \\
\hline CECA & $\mathrm{M}$ & 21 & Sim \\
\hline CECA & $\mathrm{F}$ & 21 & Não \\
\hline CECA & $\mathrm{F}$ & 30 & Não \\
\hline CCB & $\mathrm{M}$ & 28 & Sim, bastante \\
\hline CCB & $\mathrm{M}$ & 21 & Sim \\
\hline
\end{tabular}

\begin{tabular}{|l|l|l|l|}
\hline CCB & $\mathrm{F}$ & 18 & Não \\
\hline CCB & $\mathrm{F}$ & 24 & Sim \\
\hline CCE & $\mathrm{M}$ & 23 & Não \\
\hline CCE & $\mathrm{M}$ & 24 & Não \\
\hline CCE & $\mathrm{F}$ & 22 & Não, é feio para os dois \\
\hline CCE & $\mathrm{F}$ & 26 & Não \\
\hline CTU & $\mathrm{M}$ & 30 & Sim \\
\hline CTU & $\mathrm{M}$ & 30 & Não, para ambos é horrível \\
\hline CTU & $\mathrm{F}$ & 28 & Sim \\
\hline CTU & $\mathrm{F}$ & 24 & Não \\
\hline CCS & $\mathrm{M}$ & 30 & Sim \\
\hline CCS & $\mathrm{M}$ & 20 & Não \\
\hline CCS & $\mathrm{F}$ & 27 & Sim \\
\hline CCS & $\mathrm{F}$ & 26 & Sim \\
\hline CEFE & $\mathrm{M}$ & 27 & Sim, muita \\
\hline CEFE & $\mathrm{M}$ & 30 & Não \\
\hline CEFE & $\mathrm{F}$ & 30 & Não, é tudo horrível \\
\hline CEFE & $\mathrm{F}$ & 29 & Sim \\
\hline
\end{tabular}

Recebido em: junho de 2020 . Aprovado em: agosto de 2020.

\section{Como citar este trabalho:}

VIANA, F. C.; ALVES, J. B. O uso de gírias: crenças, preconceitos e identidades.

Traços de Linguagem, v. 4, n. 1, p. 104-112, 2020. 